\title{
ANÁLISIS DEL IMPACTO DEL ÁREA DE TECNOLOGÍA EN EL CICLO BÁSICO Y SU REPERCUSIÓN EN LAS INSTANCIAS DE EVALUACIÓN DE LA ASIGNATURA SISTEMAS ESTRUCTURALES I
}

Eje 3: Extensión universitaria y transferencia tecnológica

\author{
Máster Bruschini Arq. César ${ }^{1}$ \\ Arq. Armelini Griselda R. ${ }^{2}$ \\ Arq. Herrero Valeria ${ }^{3}$ \\ Arq. Feigielson Sofía ${ }^{4}$ \\ TC Godoy Yaín A. ${ }^{5}$
}

Estud. Bruschini Lucía ${ }^{6}$

${ }^{1}$ Facultad de Arquitectura, Diseño y Urbanismo - Universidad Nacional del Litoral, Santa Fe, Argentina, bruschin@fadu.unl.edu.ar

${ }^{2}$ Facultad de Arquitectura, Diseño y Urbanismo - Universidad Nacional del Litoral, Santa Fe, Argentina, griarme@gmail.com

${ }^{3}$ Facultad de Arquitectura, Diseño y Urbanismo - Universidad Nacional del Litoral, Santa Fe, Argentina, arq.vh@hotmail.com

${ }^{4}$ Facultad de Arquitectura, Diseño y Urbanismo - Universidad Nacional del Litoral, Santa Fe, Argentina, arq.s.feigielson@gmail.com

${ }^{5}$ Facultad de Arquitectura, Diseño y Urbanismo - Universidad Nacional del Litoral, Santa Fe, Argentina, yain09@gmail.com

${ }^{6}$ Facultad de Arquitectura, Diseño y Urbanismo - Universidad Nacional del Litoral, Santa Fe, Argentina, lu.bruschini@gmail.com

\section{RESUMEN}

A través de nuestra experiencia docente dentro del ámbito educativo y de desarrollo de la asignatura Sistemas Estructurales 1 (SE1), nos vemos involucrados tanto en el aprendizaje y comprensión de las implicancias de la asignatura, como en la relación que ésta guarda con las demás asignaturas correspondientes al Área de Tecnología.

Entendemos que tal responsabilidad implica considerar la repercusión que la incorporación y relación de los contenidos de dichas disciplinas afines produce sobre los conocimientos adquiridos en las estructuras. 
En la carrera de Arquitectura, el Área de Tecnología enfoca su enseñanza al conjunto de actividades y conocimientos científicos y técnicos utilizados para el diseño, planificación y construcción de nuestro entorno, propiciando la incorporación progresiva de habilidades y destrezas que permitan identificar y formular problemas y soluciones del proyecto arquitectónico, con una adecuada planificación y optimización de recursos. La comprensión e interrelación de los diversos conocimientos de éste Área debieran constituir en sí el andamiaje en la etapa de toma de decisiones durante todo el proceso de diseño.

En este contexto, las prácticas en SE1, en tanto asignatura constitutiva del Área de Tecnología, no deben ser meras ejercitaciones del manejo de herramientas, sino que también deben lograr plasmar lo aprendido en otras materias y darle unidad al proceso de aprendizaje arquitectónico, integrando la formación tecnológica a ese proceso, materializando el objeto diseñado, analizando los aspectos funcionales, formales, plásticos y tecnológicos y vinculando en el segundo nivel los conocimientos adquiridos en las asignaturas del área que le preceden.

Durante el proceso de evaluación de los trabajos prácticos de la asignatura, son tomados los mencionados principios como criterios fundamentales para determinar el nivel de logros de cada resolución, atendiendo, en primera instancia, a la correcta resolución de las problemáticas específicas de la materia $\mathrm{y}$, complementariamente, a la capacidad de integración de conocimientos adquiridos por el alumno y la satisfactoria aplicación a un caso de estudio concreto.

El enfoque se dará en los criterios de evaluación, atendiendo a la capacidad de integración de conocimientos adquiridos del alumno en dicha instancia.

\section{PALABRAS CLAVES: ESTRUCTURAS - TECNOLOGÍA - INTERDISCIPLINA - ENSEÑANZA - EVALUACIÓN}

\section{INTRODUCCIÓN}

Actualmente, como consecuencia del vertiginoso avance que se ha dado en el desarrollo de nuevas tecnologías, la necesidad de formación en la educación tecnológica de los estudiantes de arquitectura se ve cada vez más condicionada, requiriendo una cierta interrelación, evolución y acompañamiento de las disciplinas dentro del mismo área.

Reconocemos que la tecnología por su naturaleza es un área particularmente interdisciplinar, debiendo mantener una estrecha relación con otros campos de conocimiento dentro de la carrera, relación que requiere el desarrollo de diversas habilidades intelectuales imprescindibles en el estudiante de arquitectura en su formación.

En la carrera de Arquitectura, dentro del Área de Tecnología, es posible evidenciar esa interdisciplinariedad en la interacción que se produce entre los distintos campos de conocimiento que componen dicha área, manifestándose en asignaturas cuyos conocimientos adquiridos inciden directamente en el aprendizaje de la que nos compete como docentes: Sistemas Estructurales 1. Estas asignaturas son Introducción a la Tecnología, Matemática (Taller, Básica y Aplicada), Introducción a los Medios Digitales y Construcciones 1. 
Esta pluralidad dentro del Área de Tecnología lleva a perseguir diversos objetivos, a saber: la introducción del alumno en los aspectos técnicos y tecnológicos, el reconocimiento y comprensión de los valores funcionales y expresivos de las distintas soluciones tecnológico-constructivas, la conceptualización de los diversos sistemas tecnológicos que intervienen en la obra de arquitectura y sus exigencias específicas; la referenciación de la determinación dimensional de la estructura al momento del proceso de diseño; el desarrollo del conocimiento, habilidades y destrezas propias de la complejidad del área, para determinar los diferentes aspectos relativos a la estructura, instalaciones y materialidad del diseño arquitectónico; la incorporación de los conocimientos necesarios para integrar, en el proceso de diseño, los sistemas tecnológicos, los materiales de construcción y los actores que intervienen en el multidisciplinar proceso de construir el entorno; la interpretación e implementación de los aspectos que hacen a la eficiencia (relación economíafuncionalidad) de los recursos utilizados; reconocer y evaluar críticamente la implementación de los diversos materiales en la intervención de cada etapa del diseño constructivo.

Partiendo de estas afirmaciones, el Trabajo Práctico $\mathrm{n}^{\circ} 2$-desarrollado durante el $2^{\circ}$ cuatrimestre del dictado de la asignatura- pone su foco en el desarrollo de un diseño arquitectónico integral centrado en la adecuada resolución estructural, por requerimiento propio de los contenidos de la materia, pero atendiendo a la coherencia funcional, material y constructiva, aplicando conceptos teóricos de la triada vitruviana (técnica - función - forma) que fuera origen de trabajos prácticos desde el inicio del Taller Introductorio, asignatura de ingreso a las distintas carreras de diseño de la facultad, y continuado en Introducción a la Tecnología, dictada en el primer año de la carrera específica.

\section{DESARROLLO}

\subsection{El Área de Tecnología}

El Área de Tecnología se presenta como una de las tres áreas de conocimiento en las que se divide el plan de estudio de la carrera de Arquitectura y Urbanismo. Este plan se organiza como una estructura tramada compuesta por estas tres áreas de conocimiento y tres ciclos, como estadios formativos. En conjunto, forman un sistema que coordina de forma vertical y horizontal, respectivamente, los distintos objetivos y contenidos de las asignaturas.

Los ciclos de aprendizaje refieren al Ciclo Básico (introductorio), que incluye los niveles $1^{\circ}$ y $2^{\circ}$; al Ciclo Medio (formativo), que incluye los niveles $3^{\circ}$ y $4^{\circ}$; y al Ciclo Superior (profesional), que incluye los niveles $5^{\circ}$ y $6^{\circ}$. Las áreas de conocimiento son el Área de Diseño, el Área de Ciencias Sociales y la mencionada Área de Tecnología, que abarca las asignaturas Introducción a la Tecnología, Matemática (Taller, Básica y Aplicada), Introducción a los Medios Digitales, Construcciones (1, 2, 3 y 4), Instalaciones (1, 2 y 3), Sistemas Estructurales (1, 2, 3 y 4), Organización de Obras, Taller de Práctica Profesional y Arquitectura Legal.

Estas Áreas se encuentran organizadas como una estructura en espiral en la que los conocimientos se amplían y profundizan a medida que se desarrollan los Ciclos. 


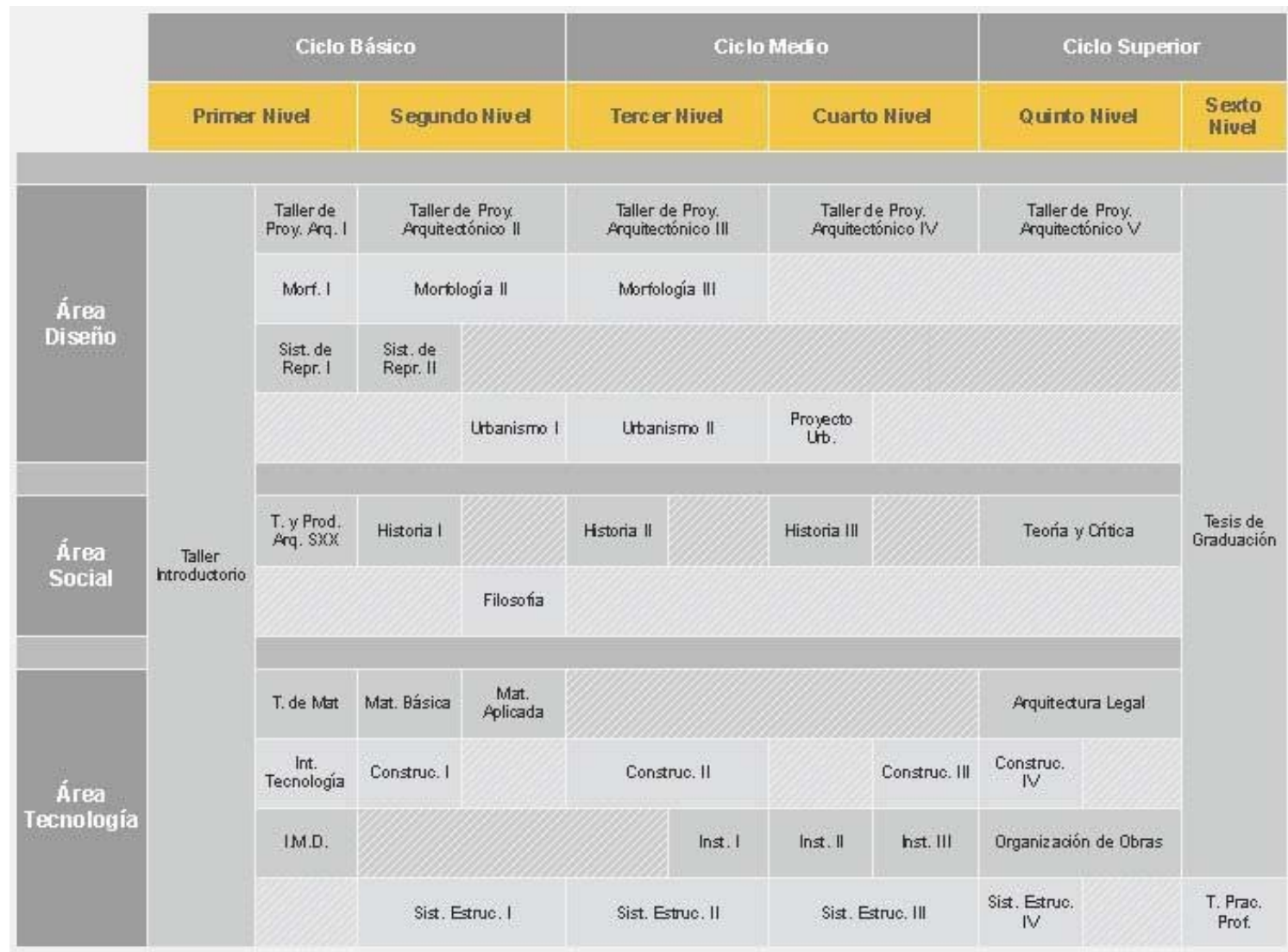

Fig. 1 - Plan de Estudio de la carrera de Arquitectura y Urbanismo.

La asignatura que nos compete, Sistemas Estructurales 1, se ubica en el denominado Ciclo Básico o Introductorio. En este se define la formación general y básica disciplinar y, entre sus principales objetivos, pretende iniciar al alumno en el conocimiento de las tres áreas de la carrera, introduciéndolo en la problemática arquitectónica, su origen, naturaleza y campos de acción; busca favorecer actividades de integración interdisciplinaria y la introducción en el manejo de los medios de representación y comunicación; y procura brindar una formación básica y general, otorgando los fundamentos necesarios para favorecer los procesos de interpretación de la realidad, de modo de generar las actitudes requeridas para el desarrollo formativo previsto curricularmente.

Para este Ciclo particular, el Área de Tecnología plantea una serie de objetivos específicos, que apuntan a que los estudiantes logren reconocer el rol de la tecnología en el diseño y procesos productivos de la construcción de la arquitectura; comprender la relación sistémica de los diferentes componentes de la obra (estructura, envolventes, instalaciones, etc.); contribuir a la formación básica del alumno que permita acceder al posterior desarrollo disciplinar; y generar las actitudes de trabajo metódico requeridas para afrontar los desarrollos específicos del área.

\subsection{Sistemas Estructurales 1}

En el desarrollo anual de la asignatura Sistemas Estructurales 1, además de perseguir los objetivos específicos del campo de conocimiento, se pretende que el alumno, a través de las instancias de evaluación, sea capaz de desplegar el conocimiento, las habilidades y las destrezas 
adquiridas en todas las asignaturas que le preceden, especialmente las contempladas dentro del Área de Tecnología, en el abordaje de la problemática de los sistemas estructurales, los sistemas constructivos y la síntesis arquitectónica, mediante el análisis de la estructura como una resolución condicionante del diseño, el estudio crítico-comparativo de diversas alternativas y la comprensión de la relación con los demás aspectos del objeto arquitectónico.

El análisis y relación de las diferentes disciplinas del área es de fundamental importancia ya que le permite al alumno utilizar las herramientas desarrolladas por el docente en las instancias teóricas, creando las condiciones para que capitalice el conocimiento adquirido.

Es sabido que los alumnos son futuros diseñadores, es por ello que las prácticas no deben ser meramente ejercitaciones del manejo de dichas herramientas, sino también instancias para lograr vincular los conocimientos adquiridos de otras asignaturas, dándole unidad al proceso de aprendizaje arquitectónico e integrando la formación tecnológica a ese proceso, con el fin de materializar el objeto diseñado, analizando los aspectos funcionales, formales, plásticos y tecnológicos.

En este marco, el Trabajo Práctico $\mathrm{n}^{\circ} 2$ contempla un nivel de complejidad media, en donde se evalúan los criterios adoptados para el ordenamiento estructural y cómo se relaciona dicha estructura con las posibilidades constructivas y funcionales.

Si bien el foco se encuentra sobre el proceso de evolución del planteo y desarrollo de la estructura y en la creatividad de la resolución de la geometría, se pretende también que el alumno demuestre la comprensión y viabilidad de los diversos recursos materiales y técnicas constructivas adoptados para cada caso.

\subsection{El Trabajo Práctico $n^{\circ} 2$.}

Luego de la introducción a los contenidos teórico-prácticos específicos de la asignatura ${ }^{1}$ y de un primer trabajo práctico de aproximación a la problemática estructural a partir del análisis de edificios construidos, el Trabajo Práctico $n^{\circ} 2$ se presenta como la primera instancia propositiva que deben realizar los alumnos.

El desarrollo de este trabajo se realiza a partir de dar solución edilicia a un Programa Funcional fijado por la cátedra. Ante este determinado Programa, el alumno debe diseñar la estructura del proyecto -cuyo único requisito es ser independiente-, proponiendo el cerramiento lateral y superior y la distribución interna en función del programa de necesidades, y determinando la tipología estructural a utilizar y su materialidad, acorde con la solicitación que corresponda en cada caso.

La pretendida integración de los conocimientos adquiridos en asignaturas previas, queda de manifiesto en la guía a utilizar por los alumnos para la resolución del Trabajo Práctico, en la que se presenta como objetivo diseñar la geometría de la envolvente espacial, localizando e identificando los componentes estructurales y de cerramiento, considerando asimismo la importancia de la interrelación que la misma guarda con los diferentes núcleos de instalaciones y la funcionalidad del proyecto de diseño en su conjunto; e interpretar la importancia del diseño

\footnotetext{
${ }^{1}$ Sistemas Estructurales 1 (SE1) es la primera asignatura del Plan de Estudios referida específicamente a la enseñanza de las estructuras en la Carrera. Si bien los alumnos son introducidos a algunos conceptos preliminares en la asignatura Introducción a la Tecnología, en SE1 incorporan conocimientos y destrezas propios de la resolución estructural.
} 
estructural en correspondencia con el empleo de los materiales de construcción, quedando delineada de este modo la tríada: forma-función-tecnología.

Es en este objetivo, en adición a la correcta resolución del sistema estructural, en el que se basa y fundamenta la evaluación de los resultados obtenidos por los grupos de trabajo en la resolución del diseño arquitectónico solicitado.

\subsection{El proceso de evaluación}

La modalidad evaluatoria se lleva a cabo con la participación activa del docente como guía, afianzando conocimientos y logros y controlando el hacer de los alumnos. En ese seguimiento, es posible evidenciar las actitudes particulares y grupales que se verán reflejadas en cortes parciales -a modo de entregas para la evaluación progresiva de los avances-, en los cuales podemos contar con un concepto general observado en el seguimiento tanto del grupo como de la formación individual del alumno.

Cada etapa del Trabajo Práctico tiene previsto un corte, una entrega, que cierra una instancia específica en el desarrollo propositivo: diseño; análisis y determinación de esfuerzos; verificación del diseño estructural, dimensionamiento y materialización de los componentes constructivos. En estos cortes es posible determinar, teniendo en cuenta el proceso, la evolución de alumno y sus inquietudes, el nivel de logros y alcances de cada grupo de los objetivos planteados.

Cada instancia se encuentra signada por una serie de requerimientos específicos de la asignatura; sin embargo, todo el proceso debe encontrarse atravesado por la coherencia resolutiva en cuanto a las distribuciones funcionales, a las resoluciones espaciales, a las posibles instalaciones y servicios y a las elecciones constructivas, no sólo de los elementos estructurales sino también de los cerramientos y terminaciones.

En el primer corte son evaluados los criterios del ordenamiento estructural, el proceso de evolución en el desarrollo y la creatividad puesta en la resolución de la geometría. En el segundo corte, el diseño de los componentes estructurales, el análisis y determinación de cargas y el correcto uso de los métodos gráficos y analíticos para determinar esfuerzos. En el tercero y último corte, se evalúa la materialización del objeto diseñado y la verificación del diseño estructural, el dimensionamiento de algunos elementos del mismo, el uso de los componentes constructivos (materiales y técnicas) y la resolución del detalle constructivo.

"Nuestra primera reflexión desde este marco, entonces, consiste en considerar que la evaluación es parte del proceso didáctico e implica para los estudiantes una toma de conciencia de los aprendizajes adquiridos $y$, para los docentes, una interpretación de las implicancias de la enseñanza en esos aprendizajes. En este sentido, la evaluación no es una última etapa ni es un proceso permanente. El lugar propicio tiene que ver con el lugar de la producción del conocimiento y la posibilidad, por parte de los docentes, de generar inferencias válidas respecto de este proceso(...)" 2 .

${ }^{2}$ Camilloni, A.; Celman, S.; Litwin, E. y Palou de Maté, M. (1998). La evaluación de los aprendizajes en el debate didáctico contemporáneo. Buenos Aires. Ed. Paidós. 
ANÁLISIS DEL IMPACTO DEL ÁREA DE TECNOLOGÍA EN EL CICLO BÁSICO Y SU REPERCUSIÓN EN LAS INSTANCIAS DE EVALUACIÓN DE LA ASIGNATURA

Trabajo Práctico Nº 2 - Ejemplo 1

Diseño de Envolvente / Determinación de Posibilidades Estructurales y Constructivas:

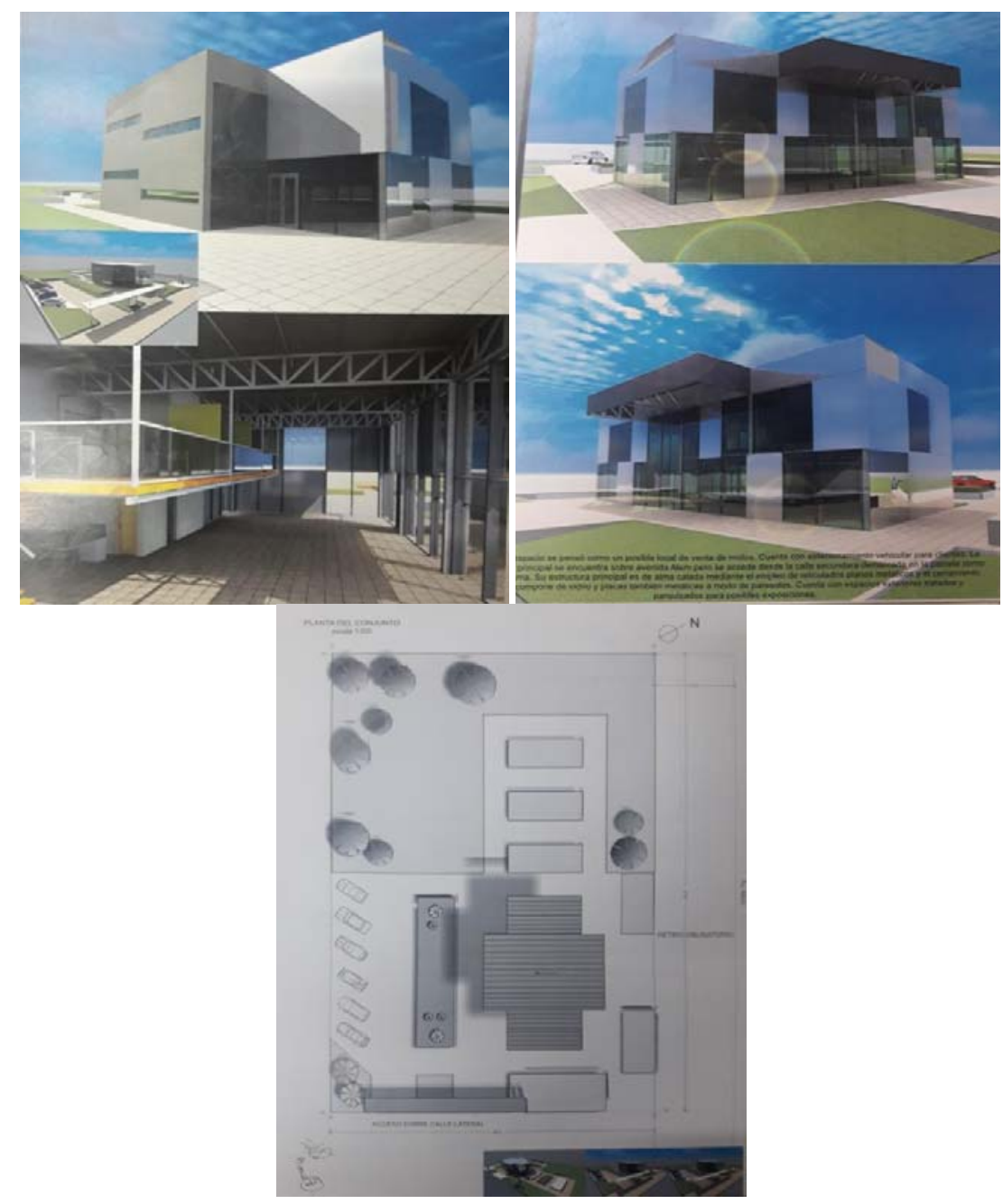




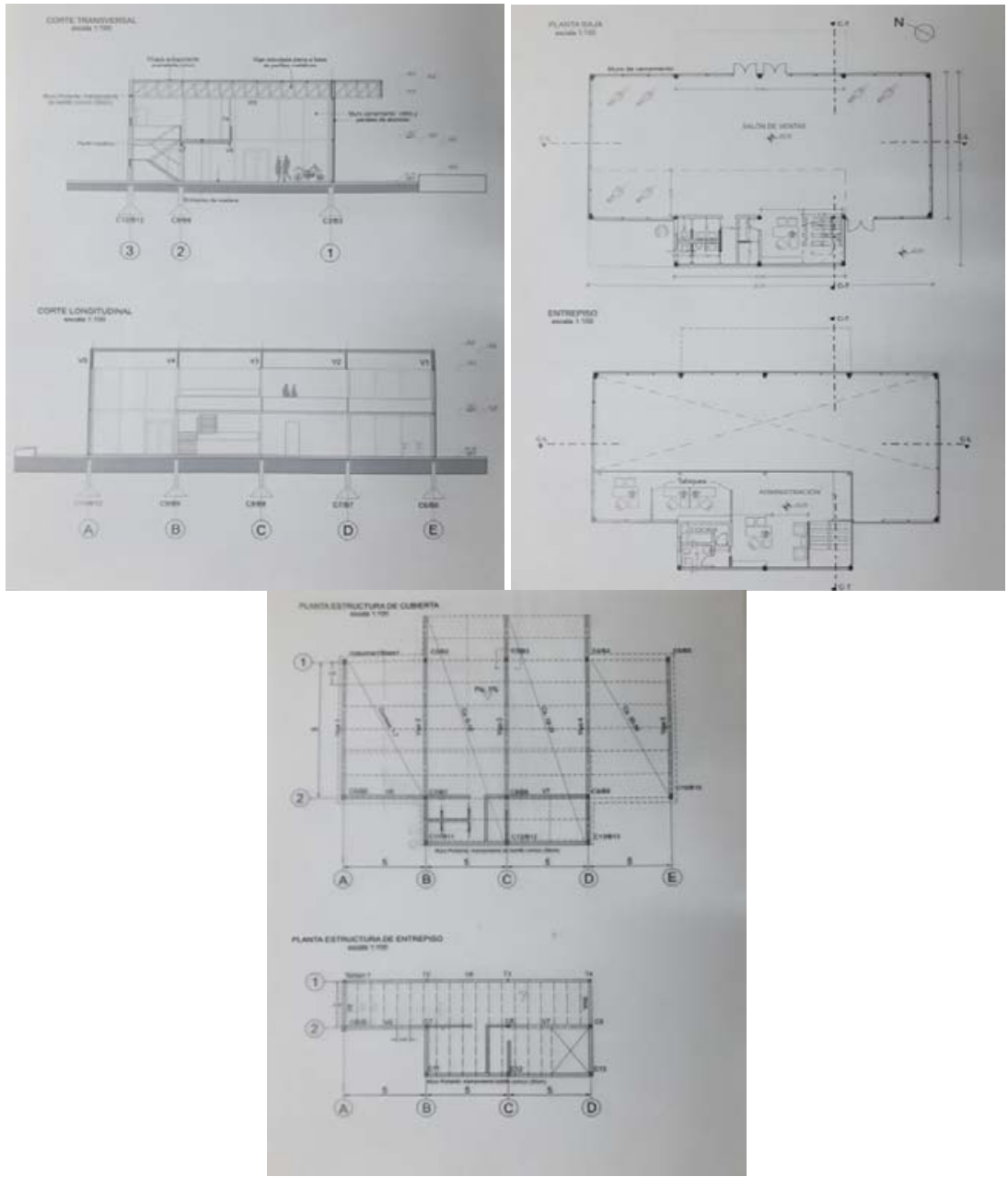

Fig. 2 a 7 - Ejemplos de Trabajos Prácticos n² 2. 
Trabajo Práctico Nº 2 - Ejemplo 2

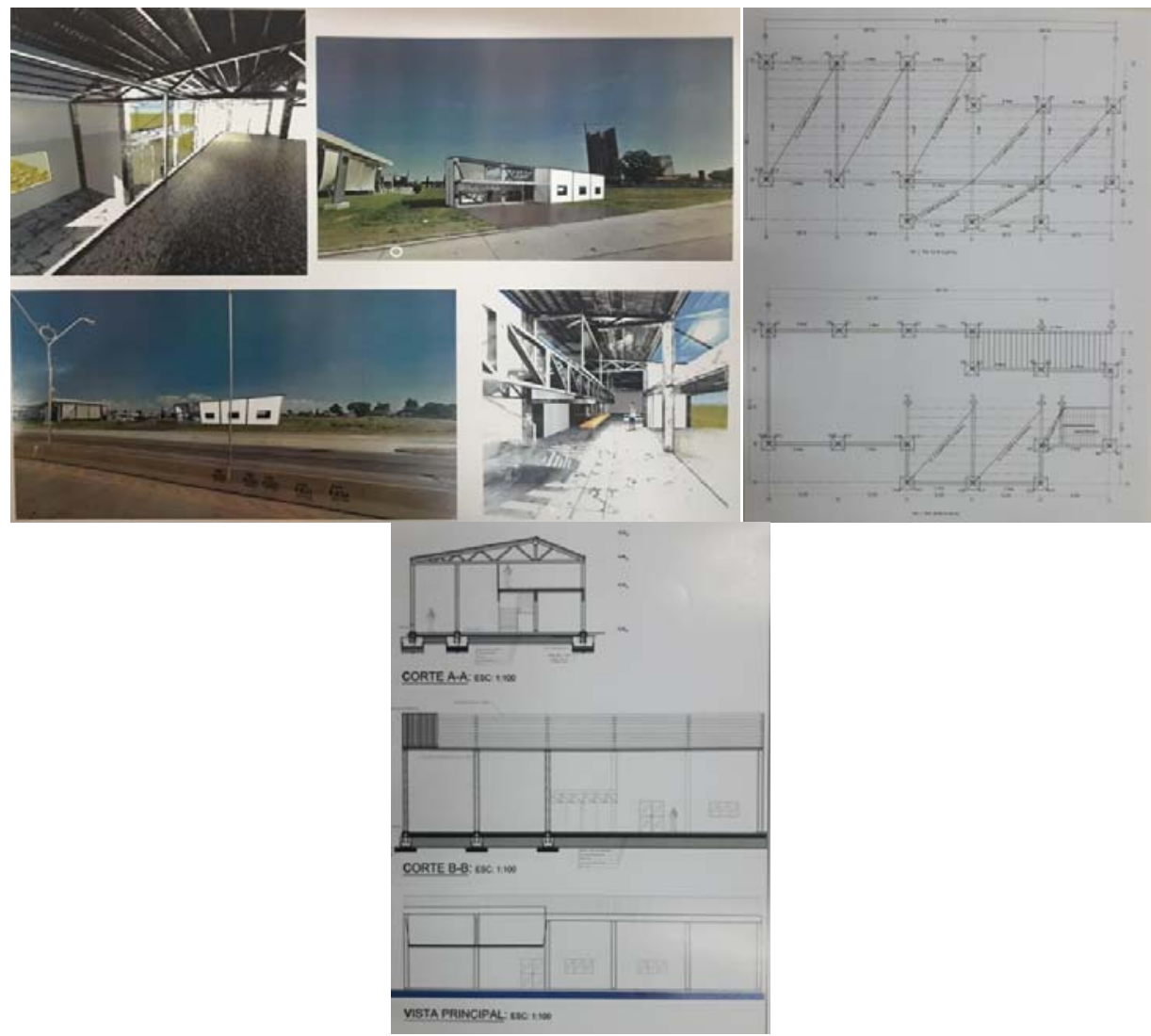

Fig. 8 a 10 - Ejemplos de Trabajos Prácticos $n^{\circ} 2$.

\section{CONCLUSIONES}

A partir de las premisas planteadas tanto para el desarrollo del Trabajo Práctico $n^{\circ} 2$ como para su evaluación, podemos afirmar que los procesos de diseño y toma de decisiones se vieron enriquecidos y potenciados por la interrelación de contenidos del Área de Tecnología y de las asignaturas afines.

Ante una problemática novedosa que implica la aplicación de nuevos conceptos para su resolución, es habitual que los alumnos recurran exclusivamente a los contenidos específicos de la asignatura y no tengan en cuenta aquellos incorporados previamente. El hecho de que en este Trabajo Práctico la integración sea una pauta explícita para la resolución de las consignas, insta a cada grupo de trabajo a abordar la problemática desde una perspectiva más amplia y les demuestra cómo esta interdisciplinariedad permite enriquecer el proceso de diseño desde la integralidad.

Los aportes de las asignaturas afines del Área de Tecnología permitieron la resolución de proyectos con adecuaciones dimensionales acordes a los Programas Funcionales requeridos, la elección de soluciones constructivas en coherencia con las particularidades de cada espacio, la definición de sus componentes con características materiales y dimensionales ajustadas a la realidad, la determinación de una distribución de locales que posibilita tanto la optimización de la estructura diseñada como la funcionalidad de los espacios e, incluso, el análisis diversos 
parámetros en congruencia con la posibilidad de futuros tendidos de instalaciones de servicio sanitarias, provisión de gas, eléctricas, pluviales-.

En cuanto a los resultados obtenidos a partir de la implementación de una estrategia evaluativa centrada en los postulados del Cognitivismo ${ }^{3}$, destacamos el doble efecto que produjo en el proceso de enseñanza-aprendizaje-evaluación. Por un lado, permitió a los estudiantes desocultar los procesos metacognitivos aplicados -consciente o inconscientemente- en la resolución de las consignas, enriqueciendo la toma de decisiones y, en consecuencia, los resultados obtenidos. Por otro lado, interpeló a los docentes a la consideración de cada saber impartido desde un enfoque integrador que amplía la visión más allá de los contenidos que competen a la asignatura, propiciando espacios para repensar las propias prácticas y convirtiendo el proceso de evaluación en una instancia de aprendizaje.

En términos de Juan Manuel Álvarez Méndez (2007), el proceso evaluativo permite al docente aprender para conocer y para mejorar la práctica en su complejidad, así como para colaborar en el aprendizaje del alumno identificando las dificultades que tiene que superar, el modo de resolverlas y las estrategias que debe implementar. El alumno, mientras tanto, aprende de y a partir de la propia evaluación y de la corrección y la información contrastada que le ofrece el docente durante las instancias de evaluación.

"En términos precisos, debe entenderse que evaluar con intención formativa no es igual a medir ni a calificar, ni tan siquiera a corregir. Evaluar tampoco es clasificar ni es examinar ni aplicar tests. (...) De estas actividades artificiales no se aprende. Respecto a ellas, la evaluación trasciende. Justo donde ellas no alcanzan, empieza la evaluación educativa. Para que ella se dé, es necesario la presencia de sujetos"4.

\section{BIBLIOGRAFÍA}

Camilloni, A.; Celman, S.; Litwin, E. y Palou de Maté, M. (1998). La evaluación de los aprendizajes en el debate didáctico contemporáneo. Buenos Aires. Ed. Paidós.

Álvarez Méndez, J.M. (2007). Evaluar para conocer, examinar para excluir. Ediciones Morata, S.L.

Bixio, C. (2003). Cómo planificar y evaluar en el aula: propuestas y ejemplos. Ed. Homo Sapiens.

Plan de Estudio de la carrera de Arquitectura y Urbanismo (2001). Facultad de Arquitectura, Diseño y Urbanismo. Universidad Nacional del Litoral.

Programa de la Asignatura Sistemas Estructurales 1 (2002), Facultad de Arquitectura, Diseño y Urbanismo. Universidad Nacional del Litoral.

\footnotetext{
${ }^{3}$ El Paradigma del Enfoque Cognitivo -teoría surgida con las corrientes pedagógicas de la Nueva Escuela- apunta a que los alumnos logren asimilar aquellos conocimientos previos que poseen y se inicien en el proceso del conocimiento a partir de la búsqueda y la investigación.

${ }^{4}$ Álvarez Méndez, J.M. (2007). Evaluar para conocer, examinar para excluir. Ediciones Morata, S.L..
} 